\section{Cahiers de Narratologie}

Analyse et théorie narratives

13 | 2006

Nouvelles approches de l'intertextualité

\title{
Narration et intertextualité, une tentative de (ré)conciliation
}

\section{Nicole Biagioli}

\section{(2) OpenEdition}

1 Journals

Édition électronique

URL : http://journals.openedition.org/narratologie/314

DOI : 10.4000/narratologie.314

ISSN : 1765-307X

Éditeur

LIRCES

\section{Référence électronique}

Nicole Biagioli, « Narration et intertextualité, une tentative de (ré)conciliation », Cahiers de Narratologie [En ligne], 13 | 2006, mis en ligne le 08 septembre 2006, consulté le 14 novembre 2019. URL : http:// journals.openedition.org/narratologie/314 ; DOI : 10.4000/narratologie.314

Ce document a été généré automatiquement le 14 novembre 2019.

\section{(c) (i) (9)}

Cahiers de Narratologie - Analyse et théorie narratives est mis à disposition selon les termes de la licence Creative Commons Attribution - Pas d'Utilisation Commerciale - Pas de Modification 4.0 International. 


\title{
Narration et intertextualité, une tentative de (ré)conciliation
}

\author{
Nicole Biagioli
}

1 Selon Antoine Compagnon ${ }^{1}$, «la notion d'intertextualité se dégage de la mort de l'auteur" advenue aux alentours des années 70 . Elle se résume pour lui dans l'affirmation de Barthes, savoureuse dans ce contexte:«le texte est un tissu de citations ». La conséquence immédiate en fut l'avènement du lecteur, ou plutôt de la lecture car, poursuit Compagnon, si « le lecteur, et non l'auteur, est le lieu où l'unité du texte se produit, «ce lecteur n'est pas plus personnel que l'auteur tout juste déboulonné, et il s'identifie lui aussi à une fonction ». C'est, nouvel rappel de Barthes, «ce quelqu'un qui tient rassemblées dans un même champ toutes les traces dont est constitué l'écrit ».

2 Compagnon aurait tout aussi bien pu écrire : «la notion d'intertextualité se dégage de "la mort de l'auteur"». Tel est en effet le titre de l'article de Barthes ${ }^{2}$ qu'il cite et commente. Par son contenu comme par sa forme, son propos est donc une invite à s'interroger sur l'évolution des relations entre l'intertexte et la narration, puisque, depuis sa mort, on le sait, l'auteur a eu le temps de ressusciter.

3 Avec le développement des recherches universitaires sur la coopération entre auteur et lecteur, les trente dernières années ont vu la recrudescence des pratiques autobiographiques, souvent interprétée comme un contrecoup du sevrage imposé par le Nouveau Roman à ses lecteurs. Personnages et auteurs se portent bien, sans parler des personnages que deviennent les auteurs racontés (par eux ou d'autres). Ont-ils pour autant chassé l'intertexte? Apparemment pas. Mais ils l'ont mis en question.

1. Du passage en revue des théories à la révision théorique

4 Le tournant du siècle se prête aux bilans; nous en avons consulté deux: celui de Compagnon, qui traite également d'autres lieux communs de la théorie littéraire, et celui, plus détaillé, de Nathalie Piégay-Gros dans son Introduction à l'intertextualité3. Nous n'y ajouterons pas le nôtre, car ils nous sont apparus à la fois suffisants et inopérants. Suffisants parce qu'ils font un point historique complet. Les deux auteurs s'entendent en gros sur trois périodes. Celle de la Nouvelle Critique, liée à l'intertexte tel que l'ont 
défini Barthes (dans l'article «Texte» de l'Encyclopaedia Universalis (1973)) ou Kristeva (Sémeiotiké, 1969) identifie la production littéraire au dialogue ininterrompu des œuvres au travers des auteurs. Les années 80 avec l'essor de la poétique restent attachées à la conception restreinte de l'intertexte défendue par Genette qui distingue l'intertextualité des autres relations transtextuelles, notamment de l'hypertextualité (Palimpsestes, 1982) et de la péritextualité (Seuils, 1987). Les années 90 se replient sur une conception moyenne, bornée à la fois par l'exigence de preuves du chercheur : il n'y en a pas partout ; la liberté du lecteur : on ne peut pas l'empêcher d'en voir là où il n'y en a pas; et l'évidence linguistique : la littérature est prise dans l'entrecroisement des discours à double titre, parce qu'elle en fait partie et parce qu'elle les répercute.

5 Si pourtant ces bilans nous ont laissée sur notre faim, c'est qu'ils obscurcissent le panorama théorique plutôt qu'ils ne l'éclairent, ce qui veut peut-être simplement dire que la période était mal choisie pour les dresser, et les conceptualisations trop mouvantes pour en arrêter la cartographie. Trois raisons au moins invitent à le croire. D'abord, ils ne parviennent pas à surmonter la contradiction interne que leur inflige le statut sémiotique «surplombant » de la littérature. Dans le principe ils reconnaissent que l'intertextualité ne peut être circonscrite à la littérature, avec une référence obligée au dialogisme bakhtinien et à la notion plus récente d'interdiscours ${ }^{4}$, comme rapport contrastif d'un discours à ceux qui l'entourent, mais, dans les faits, ils ne sortent pas du champ littéraire. Ensuite, quoique constamment préoccupés de la communication de l'intertexte, ils n'en évoquent jamais les conditions pratiques. En particulier le repérage de l'intertextualité, cheval de bataille de Riffaterre (La Production du texte, 1979), n'est envisagé que du versant de la lecture, jamais de celui de l'écriture. Enfin la relation à la narration, quoique toujours présupposée et en grande partie responsable de la restriction du phénomène intertextuel au champ littéraire et même au roman, n'est examinée que du point de vue historique, jamais du point de vue théorique.

On aurait toutefois mauvaise grâce de reprocher à des inventaires d'être ce qu'ils sont. S'ils n'évoquent ni l'intertextualité entre littérature et écrits non littéraires, ni l'interférence entre le mode énonciatif propre à l'intertextualité et les autres modes discursifs, c'est peut-être qu'elles ne saillaient pas avec assez de force des théories étudiées. Mais c'est aussi - et il ne saurait en être autrement - parce que le cadrage descriptif de l'histoire est toujours soumis à l'environnement idéologique de l'historien : on n'aperçoit du passé que ce que le présent nous y fait voir.

\section{1. Définitions}

7 Préalable à l'approche historique, ou conséquence de celle-ci, une (re)mise à plat théorique s'imposait. Pour ce faire, nous avons d'abord décidé de réduire l'intertextualité à son noyau dur, à savoir l'insertion d'un discours déjà prononcé dans un autre, dans des conditions qui préservent son identité et maintiennent le lien à la situation de discours primitive. Du strict point de vue linguistique, l'intertexte entre dans la catégorie du discours rapporté, que Patrick Chareaudeau ${ }^{5}$ définit justement comme ce qui se produit lorsque «le Propos est un Texte déjà produit par un autre locuteur " répété par le sujet parlant. Aux côtés de l'assertion, " propos qui s'impose de lui-même ", le discours rapporté constitue la troisième composante de l'organisation énonciative : la relation à l'autre tiers, les deux autres étant la relation à l'interlocuteur et la relation au dit. Cette présentation suppose deux précautions, puisque l'énonciation est à la fois un mode d'organisation spécifique et le mode d'organisation 
fondamental qui sert d'outil à tous les autres. La première est de se rappeler qu'assertion et discours rapporté se retrouvent dans toutes les organisations discursives, y compris la narration. Traitant de la citation dans la narration, nous ne nous intéresserons donc pas au discours rapporté des personnages ni au discours du narrateur sauf s'ils servent à intégrer une citation. Le seul discours rapporté qui nous concerne est la citation externe à la diégèse et empruntée au réel, et son rapporteur est la source d'énonciation effective du roman, c'est-à-dire l'auteur.

8 La seconde précaution est de prendre toute la mesure de la nature fractale de l'énonciation: le dispositif ternaire se retrouve dans la construction globale et dans chacune des composantes. Si le propos rapporté illustre une tendance de l'énonciation tournée vers le «il », il n'en est pas moins situé dans l'interlocution par rapport à un « tu » et donc relevable d'une responsabilité assumée par un «je ». On poursuivra la mise en garde de Chareaudeau, sur la prétendue objectivité du rapporteur ${ }^{6}$, en disant que si ni le rapport à l'autre ni le rapport à ce qu'on dit ne sont constitutifs de la citation, ce qui importe étant le maintien des caractéristiques de l'énoncé cité, ils l'influencent constamment.

9 Tant pour ne pas trop nous écarter du cadre originaire de la problématique que pour ne pas distendre exagérément un champ déjà pléthorique, nous avons restreint notre corpus aux récits écrits, alors qu'en principe la notion de discours rapporté est indépendante du medium. En principe, parce qu'en réalité, les propriétés physiques et structurales de l'écrit ont une incidence tant sur les pratiques citationnelles que sur les pratiques narratives, et donc sur leur interaction.

10 La distinction entre mode de discours et type de texte est fondamentale pour notre approche. C'est la présence dominante d'un mode dans un certain type de texte qui permet de le saisir. Ainsi, on a trop tendance à oublier qu'il existe en dehors des intertextes épars dans la littérature et ailleurs, un type de texte intertextuel, comme il existe un type narratif englobant roman, conte, mais aussi bien compte-rendu de presse et théatre. Ce type comporte des genres que leur projet de parole et leur situation de communication incluent dans la dominante discursive du discours rapporté. Il s'agit des recueils de proverbes, des anas, des redoutes de contrepèteries, des répertoires de charades à tiroir ou sans tiroir, des recueils de citations à l'usage des candidats au bac ou à l'agrégation, ou simplement au titre d'homme ou de femme cultivés, des anthologies, des morceaux choisis, etc. Nous n'aurons pas directement affaire à eux puisque nous n'étudions pas l'énonciation rapportée pure, mais son interaction avec la narration.

1. 2. Modèle théorique

11 Les rapports entre citation et narration ont été réduits aux cas de figure les plus simples : dominante de la narration sur la citation, puis de la citation sur la narration, et indécidabilité, c'est-à-dire relance entre les deux systèmes. Pour les décrire, nous avons fait appel à un modèle qui a fait ses preuves en mécanique, celui de la théorie du contrôle ${ }^{7}$ utilisé par exemple pour prévoir le déplacement d'un bras articulé dans l'espace: plusieurs mouvements sont nécessaires pour bouger un doigt, et il y a plusieurs possibilités pour le doigt de se déplacer. Impossible de procéder par corrélations binaires entre un point de l'espace et un « muscle » à activer. On est obligé de mettre en relation un ensemble de variables de contrôle avec un ensemble de variables d'état. 
12 Cette perspective n'est pas totalement étrangère à la narratologie. En 1972, dans Figures $3,{ }^{8}$ Genette, à la suite de Georges Blin, parlait à propos des commentaires du narrateur sur l'organisation interne du récit d'une "fonction de régie ", qui guidait le lecteur dans sa compréhension du texte. Mais implicite ou explicite, la fonction de régie des narratologues ne concerne qu'une variable de contrôle et ne la met pas vraiment en relation avec une variable d'état. Notamment, on ne s'interroge pas sur le rôle du contexte dans la réussite ou l'échec du dispositif d'élucidation. Et peut-on parler de variable quand on n'envisage pas que l'effet puisse être produit par d'autres causes, qui pourraient aussi bien être les fonctions émotive ou idéologique?

13 Le modèle du contrôle peut décrire non seulement l'ensemble des fonctionnements narratifs, (pas uniquement la métanarration), mais aussi leurs interactions: par exemple le conflit entre dévoilement de la structure et illusion narrative. Enfin il prend en charge la totalité des choix discursifs, linguistiques, médiatiques et culturels de l'émetteur : englobant donc narrateur et scripteur.

14 Le système de commande est donc ici l'auteur, et le système état, le lecteur. Il va de soi qu'il ne s'agit ni de l'auteur et du lecteur humains, ni de l'auteur et du lecteur linguistiques construits par les marques du discours, mais de modèles abstraits qui rendent compte $\mathrm{du}$ fonctionnement «inconscient» de la communication entre l'auteur- narrateur-citateur et son lecteur.

15 Dans le système auteur, nous avons placé les variables dont nous voulions comprendre la gestion : voix narrative en abscisse, reprise citationnelle en ordonnée, et dans le système lecteur leurs corrélats, jugement d'existence en abscisse et activité représentationnelle en ordonnée. Pour obtenir une analyse plus fouillée, nous avons étalonné les gradients, au moyen de termes dont on nous pardonnera, nous l'espérons, le relatif arbitraire.

16 Au sommet de la hiérarchie intertextuelle, nous avons placé la citation réinstanciée, c'est-à-dire actualisée avec tout son dispositif énonciatif (rappelons avec Schaeffer ${ }^{9}$ que la réinstanciation se distingue de l'imitation de surface par la reprise non des effets mais des actes qui les causent. Au milieu, la citation référencée, reprise du propos avec mention du nom de l'auteur, correspond au «sommaire » des narratologues, sommaire dont la citation réinstanciée serait la «scène » (cf. Genette, Figures 3, pp.130 et 140). En bas, la citation modifiée rassemble, pour reprendre la désormais traditionnelle dichotomie de Palimpsestes ${ }^{10}$, transformations et imitations.

17 S'agissant de l'identification de la voix narrative, nous avons estimé avec Käte Hamburger ${ }^{11}$ que le discours du personnage représente l'akmé de la feintise narrative, le narrateur le milieu, le scripteur le départ.

18 Côté horizon d'attente du lecteur, nous avons pensé que la fiction historique pouvait servir de moyen terme entre la vérité historique et la réalité imaginaire, pour désigner l'amalgame de faits historiques extérieurs à la diégèse et de faits inventés propre au réalisme. L'expression réalité imaginaire désigne, elle, l'assimilation des faits inventés par la noosphère.

19 Nous avons emprunté à Schaeffer (op. cit. p. 145) la notion de feintise ludique partagée pour décrire l'état parallèle qui caractérise le partenaire du jeu fictionnel, à la fois dans et en dehors de l'univers construit par le récit, ce qui nous a amenée à subsumer sous l'expression "adhésion fictionnelle » les différents types d'immersion et les leurres pré-attentionnels qui les enclenchent. Nous avons ajouté la conscience 
métacommunicationnelle, induite par les indications qui incitent le lecteur à sortir de l'univers raconté, pour « revenir à lui »...et à l'auteur. Adhésion et métacommunication sont provoquées entre autres, par ce que les pragmaticiens appellent embrayage et débrayage discursifs.

Une variable n'apparaît pas qui doit quand même être prise en compte, c'est le temps. Il va de soi - mais il est bon de le rappeler- que si citation et narration font bon ou mauvais ménage ensemble dans le texte, c'est à la faveur des enchaînements de réactions provoqués par un dispositif textuel soumis au temps et aux conditions de la lecture.

21 La progression de la description, identique dans chaque partie, reprend les cas de figure élémentaires posés à l'auteur par la gestion en simultané de la narration et de l'intertexte en croisant les deux axes :

- citation de l'intertexte avec son contexte,

23 - délégation de la citation à un narrateur,

24 - délégation à un personnage,

25 - délégation et modification.

\section{La citation narrativisée}

La relation la plus courante entre intertexte et narration, est celle de l'incorporation de la citation dans le récit. C'est également celle qui choque le moins les représentations. En effet, on imagine volontiers la citation comme un fragment, le récit comme un flux continu dans le déroulement de la lecture, ou, au repos, comme un bloc textuel.

Cette relation a donc fini par occuper tout le devant de la scène, et lorsqu'on parle de narration et d'intertextualité, c'est en général à elle, et à elle seulement, que l'on pense. On croit également que la citation fait partie du récit : il rapporte les actes et les paroles des personnages, alors pourquoi pas les actes et les paroles de l'univers extradiégétique? Or pour les différencier, il suffit de faire jouer le critère de temporalité : l'intertexte est un propos vrai, qui a été tenu, donc datable dans l'univers de référence: ce qui n'est pas le cas des propos des personnages rapportés par la diégèse.

Le schéma suivant met en regard les quatre modes de gestion de l'intertexte et de la narration dans la narration dominante (a1, a2, a3, a4) et les états (A1, A2, A3, A4) qu'ils provoquent chez le lecteur. Nous examinerons successivement la reprise historique qui met en scène les locuteurs originaux et le contexte de l'intertexte, la reprise narrative qui confie la citation au narrateur, la reprise fictionnelle qui la délègue au personnage, et la reprise hypertextuelle qui surajoute au jeu fictionnel celui de la reconnaissance sous la modification du texte et de la source originaux.

\section{1. La reprise historique}

29 La reprise historique est un récit qui, tout en dominant la citation, lui est entièrement consacré. C'est une anecdote qui restitue les circonstances de l'énonciation. Elle est indépendante du genre textuel et du mode de narration. On la trouve aussi bien dans :

30 - l'épopée avec un narrateur hétérodiégétique: «Soudain, joyeux, il dit: Grouchy! C'était Blücher » (Hugo, Les Châtiments XIII, « L'Expiation »);

31 - l'éloquence avec un narrateur homodiégétique: « $O$ nuit désastreuse! ô nuit effroyable! où retentit tout à coup, comme un éclat de tonnerre, cette étonnante nouvelle: Madame se meurt! Madame est morte! «(Bossuet, Oraison funèbre de 
Henriette -Anne d'Angleterre; la note 108 de l'édition Pléiade (1936) précise que Bossuet était au chevet de la princesse, cette nuit du 29 au 30 juin 1670); narrateur devant les énonciateurs originaux actualisés en tant que personnages, et une énonciation citationnelle non seulement fidèle mais explicative, donc au maximum de sa puissance. La tension entre pôle narratif et pôle citationnel provoque l'immersion fictionnelle du lecteur introduit au cœur de la conversation (A1), et une croyance en l'histoire due moins à la véracité du propos qu'à l'impression qu'on en est le témoin, de quoi justifier l'oxymore de " croyance historique ».

\section{2. La reprise narrative}

Si l'intégration narrative se bornait à restaurer la situation d'énonciation initiale de la citation, l'intertexte n'aurait jamais acquis la réputation de prolifération et d'exubérance qui est la sienne. La plupart des récits transforment le contexte des énoncés qu'ils citent pour les adapter à leurs besoins, qu'on peut grossièrement répartir entre racontant (gestion de la relation avec le lecteur), et raconté (gestion du contenu narratif).

Le narrateur étant une médiation énonciative, l'intertexte actualisé par le narrateur est une médiation épistémique. Sa fonction est d'arrimer l'un à l'autre les référents de l'auteur et du lecteur, opération toujours aléatoire dans une communication différée. Le narrateur peut soit la rapporter directement soit en commenter la citation confiée à un personnage, d'où sa situation intermédiaire entre narrateur et personnage (2a). Elle 
s'inscrit dans le cadre cognitif de l'intercompréhension, lequel est délimité par les quatre préconstruits (catégories minimales) du sujet, de l'espace, de l'action et du temps ${ }^{14}$. Elle procure au lecteur des informations sur celui qui lui parle, son action, sa finalité, sa localisation.

Pour cela, il faut que l'intertexte soit connu du lecteur. Le prérequis culturel explique le recours fréquent aux textes fondateurs, ainsi cette allusion biblique dans Pêcheur $d^{\prime}{ }^{\prime} s l a n d e^{15}$ : «La lumière matinale, la lumière vraie, avait fini par venir ; comme au temps de la Genèse, elle s'était séparée d'avec les ténèbres qui semblaient s'être tassées sur l'horizon».

Certains intertextes sont spécialisés. Ceux des brèves renseignent sur l'espace et l'action : les Nouvelles en trois lignes, de Félix Fénéon, publiées dans Le Matin de 1905 à 1906 annonçaient ${ }^{16}$ : "Derniers télégrammes transmis par nos fils spéciaux des bureaux du Matin à Londres et à New York aux bureaux du Matin à Paris ». D'autres sont des repères chronologiques, comme le slogan "Viva Verdi », acronyme de "Vittorio Emmanuele, re d'Italia », apparu seulement le 3 février 1901, six jours après la mort du compositeur, mais généralement antidaté de 1859, début de la seconde guerre de libération contre l'Autriche ${ }^{17}$.

41 Les plus intéressants sont ceux qui s'adressent à plusieurs publics, notamment dans le roman, parce que l'auteur doit prévoir la relation à ses contemporains et aux lectorats futurs, situation qui se complique encore lorsqu'il décrit un référent contemporain, qui l'oblige à gérer les clivages culturels et idéologiques de sa propre société. Pour Les Illusions perdues, Balzac choisit André Chénier à la fois comme révélateur des distances socio-géographico-culturelles entre Paris et la province, et comme embrayeur de rapprochement. Il en propose deux lectures. La première, privée, est faite par Lucien, et son ami imprimeur Séchard ${ }^{18}$ : "David lut, comme savent lire les poètes, l'idylle d'André Chénier intitulée Néére, puis celle du Jeune Malade, puis l'élégie sur le suicide, celle dans le goût ancien, et les deux derniers ïambes ». Elle s'adresse plutôt au lectorat contemporain. Se moquant gentiment des jeunes provinciaux, qui confondent poésie et sentimentalité ${ }^{19}$, elle ne vexe personne. La seconde, dans le salon de Mme de Bargeton, se tourne vers les générations futures. Elle exploite la particularité de Chénier, personnage commun à l'histoire de la littérature et à l'Histoire, et la fortune littéraire de l'œuvre: "Lucien avait essayé de déifier sa maîtresse dans une ode qui lui était adressée sous un titre inventé par tous les jeunes gens au sortir du collège. Cette ode, si complaisamment caressée, embellie de tout l'amour qu'il avait au cœur, lui parut la seule œuvre capable de lutter avec la poésie de Chénier ». (p. 417, nous soulignons). Jamais actualisé, peu cité, le plus souvent mentionné, l'intertexte émanant du narrateur a surtout une fonction encyclopédique. Titres des œuvres et vers retenus ne sont que des prédicats de la description de l'auteur cité. Cette encyclopédie narrative interne au récit correspond à l'idée que l'auteur se fait des connaissances de son lecteur. En général, elle aggrave le processus de métonymisation de la culture, résumant le tout (l'auteur cité) par la partie (la citation) : (« Voilà donc ce qu'est André Chénier? s'écria Lucien à plusieurs reprises»), d'où sa position médiane sur l'axe citationnel du système contrôle (a2).

$\mathrm{Au}$ lecteur construit par l'auteur réplique l'auteur construit par le lecteur. Celui-ci ne laisse rien présager des connaissances de l'auteur réel, lequel ne tire peut-être sa citation que d'un savoir d'emprunt et non de sa culture propre. Il est donc suspendu entre vérité historique (celle de la citation) et fiction historique (celle de l'auteur 
citant). Toutefois sa conception (A2) dénote une posture dialogique du lecteur qui réagit comme un partenaire en essayant de se représenter l'auteur, d'où le positionnement entre feintise ludique et conscience métacommunicationnelle.

2. 3. La reprise fictionnelle

Le personnage ne s'adressant pas à lui directement, le lecteur n'est pas vis-à-vis de lui en position d'interlocuteur, mais de spectateur. Or la fonction principale du personnage dans la diégèse n'est pas de fournir des renseignements sur l'univers décrit, sinon indirectement, mais d'incarner l'action. La citation dans sa bouche retrouve les deux fonctions propres au récit : caractérisation du personnage (description) et motivation de l'action (actantialité).

Elle remplit souvent les deux. Dans Mont-Oriol de Maupassant, Paul commence sa cour à Christiane en lui récitant les deux premières strophes de l'Hymne à la beautée de Baudelaire. Au plan descriptif, cela le situe dans un certain microcosme dandy parisien, frotté de belles-lettres mais en retard sur l'actualité littéraire. Au plan narratif, le séducteur croit tenir un adjuvant, mais Christiane est plus étonnée qu'impressionnée par l'accès de lyrisme de Paul, et celui-ci s'interrompt, piqué, désaccord prémonitoire de l'issue malheureuse du roman, ce qui en fait un opposant.

Investie dans la représentation de l'action, la citation cesse de jouer son rôle de trace mnésique ${ }^{21}$, de dénoter, pour connoter. Elle connote d'abord sa source, qui peut être le sociolecte ambiant « les femmes, furieuses de ne pas avoir de poète à leur service pour les traiter d'anges, se levèrent comme ennuyées, en murmurant d'un air glacial : très bien, joli, parfait " (Les Illusions perdues, p. 417), comme l'idiolecte d'un auteur : Chénier devient du Chénier, Baudelaire du Baudelaire. Elle connote aussi son contenu : l'amour et la sentimentalité, communs à la plupart des poèmes. Elle connote aussi le signifiant : la citation poétique connote la poésie, voire l'art dans son ensemble. Dans Mont-Oriol, Paul rapproche le poème de Baudelaire de La Joconde. Ces connotations sont des connotations culturelles attachées aux interdiscours. La généralisation qui en résulte permet ensuite à la citation de passer à la connotation narrative, celle qui caractérise un personnage ou symbolise un enjeu actantiel.

Il serait facile de conclure que les personnages citants dans un récit représentent les discours sociaux auxquels leurs citations sont empruntées. C'est une constante du roman du $19^{\text {ème }}$ et même du $20^{\text {ème }}$ siècle (que l'on pense à Sarraute ou à Pinget) que de vouloir et de parvenir à représenter des interactions verbales. Pourtant, cette représentation passe toujours par une appropriation qui évite au personnage de tomber dans le stéréotype, sauf, bien entendu, si on veut montrer qu'il est un stéréotype, et, même dans ce cas, le stéréotype est personnalisé.

Dénonciation ou représentation, le personnage citateur apparaît donc comme un produit du discours social qui le traverse. Pour l'auteur, il représente un exercice d'exorcisation (a3) l'occasion de s'affranchir des discours sociaux, au moins en les dominant par le jeu de la représentation. Pour le lecteur, la compétence citationnelle attribuée au personnage est un rappel de l'univers de référence, qui, certes, produit un effet de réel, mais maintient aussi son lien avec l'Histoire, d'où sa place entre fiction historique et réalité imaginaire (A3). Elle produit une autoreprésentation du mécanisme citationnel (le personnage reproduisant l'acte qui est en réalité celui de l'auteur), sans pour autant faire accéder le lecteur à l'activité du scripteur, donc à michemin entre feintise ludique et conscience métacommunicationnelle. Citation du narrateur et citation du personnage ont la même provenance : le stock culturel choisi 
et distribué par l'auteur sur les différents niveaux narratifs. C'est pourquoi le personnage citateur renvoie au lecteur son propre reflet de locuteur. Le lecteur construit (a2) était déterminé par le choix des citations assumées par le narrateur, le double citant du lecteur (A3) est constitué non par un personnage citateur particulier, mais par l'ensemble des citations déléguées aux personnages, puisque le lecteur est, par rapport à elles en position non plus de récepteur direct, comme il l'était face au narrateur, mais d'acteur qui prête sa voix. Si l'auteur construit exerçait la capacité du lecteur à imaginer son partenaire, le double citationnel l'invite à interpréter dans le récit une partition qu'il connaît bien : celle du polylogue social.

\section{4. La reprise hypertextuelle}

49 La modification de l'énoncé citationnel (a4) suppose, pour être appréciée, que le lecteur puisse retrouver l'hypotexte sous l'hypertexte. Le dévoilement peut avoir lieu avant la modification, ce qui advient lorsqu'on utilise des textes très connus, notamment pour la parodie, ou après : si l'on restitue un plagiat à son véritable signataire. Dans tous les cas, la perception de l'écart détourne immanquablement l'attention du narrataire de la représentation. L'effet produit sur le lecteur (A4) se range donc d'emblée du côté de la conscience métacommunicationnelle.

Reprenant la classification de Genette dans Palimpsestes nous rangerons les hypertextes d'après l'opération qui les constituent: transformations (parodies, oulipismes) d'un côté, imitations (plagiats, textes d'école, pastiches) de l'autre. Non sans remarquer par anticipation que cette diversité des moyens concourt à un but unique, qui est de rapprocher le lecteur de l'auteur cité. Au naturel, les hypertextes ont déjà cette fonction de médiateurs culturels, rendant leurs hypotextes plus familiers, aux deux sens du terme : mieux connus, moins vénérés. Ils la conservent dans le récit.

51 Les imitations ne font que pousser à l'extrême les conséquences du changement de statut sémiotique de la citation dans le récit. Puisque l'acclimatation de la citation authentique oblige à une double transformation, de la dénotation à la connotation culturelle, puis de celle-ci à la connotation narrative, il est plus simple de fabriquer une citation ad hoc, en imitant les discours sociaux tout en tenant compte du contexte narratif. On trouve dans Mont-Oriol, à côté des citations vraies, de nombreux pastiches de genres: le prospectus de la ville de cure, l'affiche du casino, l'ordonnance du médecin, l'acte de vente du champ où est située la source thermale.

52 Le plagiat s'explique de même par la capacité des textes fortement stéréotypés (ceux dont le plagiat est le moins risqué) à connoter leur époque plutôt qu'à dénoter leur auteur. Encore une valeur toute prête. La meilleure preuve, c'est que la restitution ne change rien à leur fonctionnement représentatif. Balzac n'a pas hésité à faire endosser à Lucien certaines de ses œuvres de jeunesse ${ }^{22}$. Les poèmes avec lequel Lucien espère conquérir la gloire: Les marguerites ${ }^{23}$, ont été commandés à des amis. Le second, de Delphine de Girardin, le premier et le troisième, de Charles Lassailly, ressemblent à ce qu'ils étaient pour l'époque, des œuvres d'école. La tradition veut qu'il ait gardé le meilleur, celui de Gautier, pour la fin. Mais si Lucien le trouve meilleur que les autres, rien ne prouve que c'était l'avis de Balzac. Le narrateur se borne à souligner que, sur ce point aussi, le héros manque de personnalité, se fie au goût, et aux formules, d'autrui : "c'était le sonnet que préféraient d'Arthez et Brideau, peut-être à cause de sa couleur».

53 Le lecteur, lui, n'y voit que...des fleurs, d'autant que le premier vers «Moi, je suis la tulipe, une fleur de Hollande", ressemble étrangement à celui de Delphine de 
Girardin : «Je suis la marguerite et j'étais la plus belle». Au bout du compte la fonction romanesque l'emporte sur la valeur esthétique et le poème de Gautier paraît tel que Balzacl'a voulu: à peine meilleur que ceux des autres. Ainsi les imitations citationnelles dessinent-elles une parfaite image du discours social, mais sans le relier à des circonstances énonciatives précises : une prime à la banalité.

Les transformations parviennent au même résultat par des voies opposées, puisqu'elles conservent la source. Astérix chez les Belges ${ }^{24}$ qui traite comme tous les albums de la série de Goscinny-Uderzo, de la Guerre des Gaules, offre une panoplie complète de parodies et de travestissements burlesques. Ceux-ci frappent alternativement les citations d'auteurs contemporains (Trenet: « je chante, je chante tout en latin, je chante, je suis romain » p. 7 ; Brel: « dans ce plat pays qui est le mien, nous n'avons que des oppidums pour uniques montagnes » p. 20), et les citations historiques (dépossédé de la version originale d'une de ses célèbres citations - son aide de camp se permet de dire «alea jacta est » avant la bataille, p. 39, César récupère la seconde, mais en français et au futur : «j'irai, je verrai et je vaincrai », p. 30). L'anachronisme discursif suggère que les Romains, même les plus illustres, n'étaient guère différents des Français actuels.

La médiation culturelle ne s'arrête pas là car la cible du travestissement est moins le sujet noble: ici l'épisode historique, que le genre sérieux dont le sujet n'est qu'un composant : l'épopée. Le récit de bataille (p. 42 sqq.) parodie la description de Waterloo dans Les Châtiments ${ }^{25}$ : «Le soir tombait; la lutte était ardente et noire /César avait l'offensive et presque la victoire ; /Il tenait les Belges acculés sur un bois ».

La modification de l'intertexte trahit l'activité scripturale au double sens du verbe : elle la rend apparente grâce à l'écart entre hypo- et hypertextes, mais la cache derrière la connivence culturelle, son but principal. La réalité historique est décomposée en référence et en vécu, ce qui cible la place de la fiction historique. Cette démystification (A4) rapproche l'univers de la fiction de celui du lecteur, mais pas les personnages. Dévalués, caricaturés, ceux-ci font tomber l'adhésion fictionnelle à son point le plus bas.

57 Issu de l'univers de référence des locuteurs, l'intertexte est un intrus que le récit s'efforce de capturer à son profit en limitant autant que faire se peut ses effets "dégrisants ». Depuis Bakthine, on a souvent débattu de la difficulté à délimiter les concepts d'intertexte et d'interdiscours. Si l'on définit l'interdiscours comme la force centripète qui groupe les énoncés autour de problématiques conversationnelles ritualisées ou institutionnalisées, et l'intertextualité comme la force centrifuge qui attire les énoncés vers des emplois différents de leurs emplois habituels, on s'aperçoit que le récit n'emprunte l'intertexte que pour lui faire représenter son contexte. Il ne faut donc pas confondre l'interdiscours réel source de l'intertexte et l'interdiscours représenté par le récit. La narration convertit l'indice en icône, avec comme conséquences la narcotisation de la différence fiction/réalité dans les discours cités, et l'impossibilité de faire apparaître la nature de l'opération intertextuelle même en la déformant. Toutefois la présence des interdiscours matérialisés par l'intertexte maintient à l'intérieur de la structure fermée du récit des fenêtres ouvertes sur le monde, laissant présager qu'à son tour celui-ci pourra devenir matière à citation, qu'il n'est, lui aussi, que discours.

3. Le récit intertextuel

58 L'écriture intertextuelle est l'écriture des « ciseaux et [du] pot à colle ${ }^{26}$. Elle n'est pas neuve. Dans l'Antiquité, Ausone avait composé un centon nuptial assez leste avec des 
vers de Virgile. Nous ne traiterons ici que des collages qui prennent la forme d'un récit. Celui-ci est-il construit par le lecteur ou prévu par l'auteur dès le choix des morceaux ? Question de lectures : celle du lecteur prompt à «broder " à partir de la première information venue, celle de l'écrivain, capable de déceler les potentialités narratives du moindre fragment linguistique.

Le schéma suivant met en regard les quatre modes de commande de l'intertexte et de la narration dans la narration intertextuelle (b1, b2, b3, b4) et les états (B1, B2, B3, B4) qu'ils provoquent chez le lecteur.

Reconduisant le schéma de la première étude, nous examinerons successivement le centon éditorial qui juxtapose des citations conservant leurs locuteurs et leurs contextes, le centon narratif qui confie à un narrateur le montage des éclats intertextuels, le centon fictionnel qui met en scène les locuteurs authentiques dans une situation nouvelle, et le centon hypertextuel qui superpose au jeu fictionnel celui de la reconnaissance de l'hypotexte.

\section{1 . Le centon éditorial}

61 Il arrive que des textes déjà communiqués soient repris dans un autre cadre, comme la publication d'une correspondance privée, avec un autre objectif, comme la réalisation d'une biographie, ou dans une autre version (J. B. Pontalis couple la Correspondance de Lou Andreas-Salomé et Sigmund Freud (1912-1936) avec le Journal d'une année de celle-ci, parce qu'il s'agit de l'année 1912-13) ${ }^{27}$. Ce qui fait la reprise est donc le décalage temporel (datées de 1820-22, les Lettres à la fiancée de Hugo ne sont publiées qu'en 1901), et l'intervention d'un éditeur. Réputée subalterne, la reprise éditoriale est une réécriture qui n'est ni négligeable ni anodine. L'éditeur est responsable du choix des textes, de leur ordre. Et s'il n'ajoute rien dedans, il se rattrape dans le péritexte.

Le premier échange épistolaire entre Lou Salomé et Freud (3 et 4 -11-1912) contient déjà un épisode et deux schémas actantiels contradictoires. A peine Lou fait-elle la connaissance de Freud qu'elle se trouve tiraillée entre les pères ennemis de la psychanalyse, Adler et Freud. La requête de ce dernier : "Je vous demande seulement, eu égard à la situation, et comme pour un clivage psychique artificiel, de ne faire aucune mention de votre existence ici et réciproquement » (op. cit., p. 13), esquisse un provisoire retour à l'équilibre.

Mais où est le narrateur? Genette ${ }^{28}$ classe les types de préfaces selon l'identité des préfaciers: allographe/autographe; actorial/auctorial, fictionnel/fictif. Il nous a semblé plus rentable de considérer que certaines préfaces éditoriales ne font que commenter une construction narrative implicite, et que le préfacier était en fait un narrateur. Elles sont donc allographes hétérodiégétiques, ou autographes, écrites par un des locuteurs cités par la reprise, avec un narrateur homodiégétique, si ce n'est pas le locuteur principal (en général le plus célèbre), ou autodiégétique.

Pourtant le mode narratif est aussi indifférent dans la narration intertextuelle qu'il l'était dans l'intégration narrative. Ce qui fait la différence, c'est le degré et la nature de l'intervention de l'arrangeur. Pour justifier la macro-phrase du titre «X par lui-même » la maquette éditoriale des "Ecrivains de toujours » exige qu'une surface considérable du texte soit occupée par des citations, souvent sans solution de continuité. Souvent, mais pas toujours. Le biographe remplit alors les vides par des adjonctions narratives. Les suppressions ne sont pas moins significatives. Ainsi la décision de J. B. Pontalis de regrouper en fin de volume plutôt qu'en bas de page les notes du premier éditeur de la 
correspondance de Lou Salomé avec Freud n'est pas étrangère à son attrait romanesque.

Le deuxième cas d'oubli décrit par Marc Augé (op. cit., p. 76) est la " possession » qui tend, en laissant occuper la conscience par une présence étrangère, «à retrouver le présent en le coupant provisoirement du passé et du futur ". C'est exactement ce à quoi consent l'arrangeur. Il semble s'effacer complètement pour laisser parler d'autres voix, et cette transe (b1) impressionne. Gide confiait que la correspondance de Flaubert avait «durant plus de cinq ans, à [s] on chevet remplacé la Bible ${ }^{29}$. Toutefois le péritexte est là pour contenir la transe dans le présent de la lecture. Il nous rappelle que sans le « medium » arrangeur, nous ne pourrions pas entendre la voix de l'auteur cité. On reste donc dans la feintise ludique partagée, au plus près de la vérité historique (B1).

\section{2. Le centon narratif}

en scène les absents, les morts, les êtres surnaturels, ou même les êtres inanimés, pour les faire agir, parler, répondre », telle est la définition de la prosopopée ${ }^{30}$. A cet objectif pragmatique, la reprise citationnelle ajoute une contrainte textuelle : elle ne met dans la bouche du locuteur fantasmé que des propos qu'il a vraiment tenus, limitant l'intervention du narrateur au péritexte. La fausse interview, tout en conservant la contrainte du centon, lève la contrainte d'implicitation. Le narrateur apparaît au début, pour rappeler les conditions de l'interview et dans le discours rapporté, puisqu'il est un des protagonistes. Bernard Mérigaud débute son « entretien exclusif avec Verdi ${ }^{11} »$ (en 2001, rappelons-le) par une pseudo-narration hétérodiégétique : "Sur la route qui mène à la propriété de Verdi, l'envoyé spécial d'Opérama fredonnait crânement le fameux «Va pensiero » de Nabucco en guise d'exhortation à surmonter un trac panique », avant de se dévoiler : "...On me fit patienter[...]. Giuseppina Strepponi, la femme du compositeur, m'introduisit.. .» et de commencer l'entretien : "J'ai cru apercevoir que vous étiez en train de travailler sur un nouvel opéra...».

Interview authentique et fausse interview sont toutes deux des réécritures. Mais l'une transcrit les notes prises lors d'un échange oral, l'autre reconstitue un faux oral à partir de bribes d'écrits. Les questions conditionnent les réponses dans l'une, alors que dans l'autre elles en dépendent. En effet, elles ne servent qu'à sertir les citations dont elles respectent jusqu'à la forme grammaticale, ce qui les place dans le champ de la mise en scène citationnelle (b2).

Les leurres pré-attentionnels : accroches, intertitres, iconographie abondante légendée au présent, assurent l'adhésion fictionnelle. Mais celle-ci voit son influence compensée par :

69 - la connaissance de l'univers de référence (on sait, puisque c'est un numéro commémoratif, que Verdi est mort cent ans avant la rédaction de l'article),

70 - les marques énonciatives du narrateur (le nom du journal fictif «Opérama » calqué sur le vrai, le vocabulaire et le registre de langue, ceux d'un journaliste de la presse musicale du début du $21^{\text {ème }}$ siècle) ;

71 - le péritexte final, en petits caractères, mais essentiel ${ }^{32}$.

72 L'adhésion fictionnelle existe, mais tenue en lisière par la conscience métacommunicationnelle: dans un juste milieu (B2). Le péritexte donne les moyens d'apprécier le rapport entre la vérité historique et la situation imaginée à partir d'elle, ce qui cible l'axe de la fiction historique. 


\section{3. Le centon fictionnel}

73 L'arrangeur fait parler le locuteur de la citation, l'interviewer lui parle, l'auteur du dialogue imaginaire le fait parler avec d'autres, tout aussi absents ou morts que lui. Ce type de proposopée nous renvoie à l'Antiquité, avec Le dialogue des morts de Lucien. Les personnages n'y reprennent pas forcément leurs propos à la lettre, d'autant qu'ils sont en général convoqués pour donner leur avis sur un sujet d'actualité (celle de l'auteur, pas la leur).

Quand s'y surajoute la contrainte intertextuelle, le choix des textes devient plus délicat que pour l'interview. Bâtir un échange complet suppose de croiser les corpus des différents locuteurs. Il faut trouver des thèmes de désaccord à différents niveaux : personnels, sociaux et culturels afin de ménager des paliers de médiation; mais aussi des thèmes d'entente; et pour rythmer la conversation, des formules d'engagement, de réplique, de relance et de conclusion. C'est ce qu'a fait Catherine Vigourt qui propose, toujours dans le même numéro de Télérama, (pp. 44-46) un scoop : "Verdi-Wagner la rencontre ». Les musiciens ne se sont jamais rencontrés. Leur rivalité est un topo de l'histoire de la musique. Les répliques acerbes ne manquaient donc pas. Comme thèmes d'affrontement, on trouve, à côté de la musique, les femmes (« Wagner : vos femmes ne sont capables que des voluptés du corps! Verdi : Les vôtres sont des soldats de votre philosophie »). Les deux adversaires se rejoignent sur l'ingratitude des politiques et le succès immérité de Meyerbeer.

La première note nous prévient que « ce dialogue entre Verdi et Wagner est une fiction mais reprend des citations des deux musiciens ». Toutefois l'impossibilité historique perturbe la référence de la citation, non du point de vue de l'attribution, mais de celui du contexte : ils ont certes dit cela, mais jamais l'un à l'autre. On sort donc du champ de la référence exacte (b3).

Elle perturbe aussi la vraisemblance de la fiction, mais cela ne signifie pas qu'elle empêche l'adhésion fictionnelle. De fait, nous rencontrons tous les leurres préattentionnels du théâtre, et cet intermède pourrait très bien être porté à la scène.

Le véritable responsable de l'abaissement de l'adhésion fictionnelle est l'objectif pragmatique de ce type de fiction : montrer ce qu'il aurait pu être, et non, comme la fausse interview, ce qu'il a été. Il la condamne à argumenter, dans l'accroche : « Ils sont nés la même année et incarnent, chacun à sa façon, l'esprit d'une jeune nation européenne en train de se construire. Pourtant Verdi et Wagner ne s'entendent sur rien... » mais aussi dans le dialogue qui est une illustration de l'hypothèse : " si Verdi et Wagner s'étaient rencontrés ». L'attention du lecteur se préoccupe moins du dialogue imaginaire, que de sa plausibilité. Cette conduite évaluative relève de la conscience métacommunicationnelle. Comparant la vérité historique et la réalité imaginaire qui en a été tirée, elle redécouvre la production de la fiction historique (B3).

3. 4 . Le centon hypertextuel

Lorsque l'écriture intertextuelle intervient sur des fragments modifiés, la reconnaissance est évidemment plus difficile. Au jeu habituel des hypothèses lectorales que la suite du récit infirme ou confirme, se surajoute celui du puzzle. Il s'agit d'identifier chaque pièce et de comprendre son fonctionnement dans la nouvelle structure. Ceci vaut pour les transformations comme les imitations.

Les Propos détachés de Sam Weller d'Alphonse Allais ${ }^{33}$ appartiennent aux premières. Allais transpose des proverbes ou des expressions au moyen de la formule consacrée « comme 
disait » dans une énonciation de fantaisie aboutissant soit à une parodie (sujet bas, style noble) : «Que vouliez-vous qu'il fit contre trois? " comme disait la veuve d'un monsieur trépassé à la suite d'une consultation des trois meilleurs médecins de Paris »; soit à un travestissement burlesque (style bas, sujet noble) : «Oh! l'éternel féminin... comme disait le monsieur dont la belle-mère n'en finissait pas de claquer ». S'y ajoutent souvent des double sens : "Voilà un ouvrage qu'il faut savoir lire entre les lignes, comme disait Gyp en offrant le "Journal d'un philosophe " à un aiguilleur de la station d'Achères » et des calembours: "l'arbi traire, comme disait le poète observateur et calembourgeois, témoin des effroyables exactions de l'administration dans nos possessions d'Afrique ».

La première des procédures imitatives est le plagiat, emprunt non déclaré, dans lequel l'imitation consiste à faire croire que l'auteur original est celui qui signe le texte. Yak Rivais présente dans un même volume les deux versions de ses Demoiselles d'A. ${ }^{34}$, en annonçant d'emblée qu'elles «se composent de 750 citations d'auteurs, à raison d'une seule phrase citée [...] par ouvrage ».. La version non déclarée se lit comme n'importe quel roman. On y reconnaît les modes littéraires de l'époque: sexe et guerre, confrontations violentes des classes sociales dans la politique, le lexique et le discours. La seconde rétablit les références.

81 Le puzzle de pastiches peut être tout simplement un roman par lettres, mais Antoine Bello, dans son Eloge de la pièce manquante ${ }^{35}$, élargit le champ des genres empruntés aux articles de journaux, compte rendus de séances de sociétés savantes, et autres extraits de traités de puzzlologie. Le principe reste le même. Pas de narration explicite (le coupable ne se dévoile qu'hors récit, à la fin); juste une série d'extraits, dont la seule différence avec la reprise éditoriale est qu'ils sont faux, c'est-à-dire fabriqués à partir de discours réels.

La supercherie fait passer le faux pour du vrai, ce qui n'est pas le cas du roman. C'est ce qu'a réussi Claude Bonnefoy, en consacrant le centième numéro des Ecrivains de toujours à un écrivain imaginaire ${ }^{36}$. Maquette habituelle donc: X par lui-même, mais avec des faux.

Dans tous ces hypertextes, des leurres préattentionnels aident le lecteur à adhérer à la fiction qu'on lui propose. Les citations sont reconnaissables, les discours plagiés ou imités vraisemblables (on le vérifie si on juxtapose aux échantillons de poésie et de prose de Marc Ronceraille respectivement des extraits de Compacts de Maurice Roche (1967) et de l'Inquisitoire de Robert Pinget (1962). Le péritexte, couverture et titre, incite à une lecture normale.

Ils ne sont là cependant que pour corser le jeu littéraire. Le plagiat de Rivais est éphémère, puisque dès la couverture, le sous-titre accole roman et citations. La supercherie de Bonnefoy ne dure pas davantage, si l'on repère le calembour du titre : "l'on se raille». Elle ne survit pas à la lecture de la bibliographie " hénaurme». La réalité imaginaire (B4) s'impose, avec son mode de fabrication.

Associer la prosopopée au centon affaiblit sa parenté avec l'hallucination. Ce qui a déjà été dit peut revenir mais on sait qu'il vient d'ailleurs. Cependant cette forme de fiction témoigne du pouvoir de fascination de l'intertexte, qui semble engendrer à la fois le mal : tarissement de l'imagination narrative, et sa panacée : élaboration du récit à partir de textes existants. C'est une pratique éminemment littéraire, même si elle englobe des productions journalistiques ou philosophiques, qui suppose une solide connaissance des contextes citationnels. L'image de l'intertextualité qu'elle véhicule est paradoxalement à la fois fermée, spécialisée, et interactive. Borgésienne, elle parie que 
par dérives successives, n'importe qui peut retrouver n'importe quelle forme en partant de n'importe quel contenu.

4. Le tissage textuel

86 A côté de l'intégration de la citation par le récit et de la reconstitution du récit par la citation, il y a place pour une troisième démarche qui est l'exploitation des capacités d'échangeur sémantique et sémiotique de la citation. En effet, l'intertexte ne se borne pas à mettre en rapport deux énoncés, deux énonciateurs et deux contextes. L'adaptation de la citation au nouveau contexte exige une analyse et une manipulation des paramètres textuels. Elle développe forcément des compétences de réécriture (orale aussi bien qu'écrite). La première option entraînait la réécriture intuitivement, la seconde relevait au contraire d'une écriture de laboratoire. La troisième utilise la citation comme embrayeur de créativité et croise, ou tisse (pour rester fidèle à la figure étymologique) dans le texte l'inter- et l'intratextualités.

87 Le schéma suivant met en regard les quatre modes de commande de la citation et de la narration dans le tissage textuel (c1, c2, c3, c4) et les états (C1, C2, C3, C4) correspondants.

88 Nous passerons en revue successivement la relance énonciative dans laquelle l'auteur de la citation revit son énonciation en s'en dissociant, la relance narrative dans laquelle le narrateur utilise la citation comme embrayeur narratif, la relance fictionnelle qui met en scène l'activité citationnelle à travers un personnage, et la relance hypertextuelle qui utilise la modification de la citation comme embrayeur scriptural.

4. 1 La relance énonciative

Le troisième cas d'oubli déterminé par Marc Augé (op. cit. p. 78) est le recommencement qui consiste à « retrouver le futur en oubliant le passé, créant les conditions d'une nouvelle naissance». S'il est créatif du point de vue social, disséminant les propos loin de leur champ de discours, l'intertexte est contraignant pour son auteur. Juguler la dérive interprétative de leurs commentateurs est une préoccupation des professionnels de la parole orale ou écrite.

90 Mais « reprendre » au sens figuré et au sens propre sa parole, c'est à la fois voler ce qui n'est plus à nous mais à l'espace interlocutif, et tomber dans l'illusion de la cohérence autobiographique. Point n'est besoin de lire Montaigne pour constater que d'un moment à l'autre «Je » ne se ressemble pas. C'est donc moins à se reprendre qu'à se déprendre que le cité soucieux de ses dires doit s'efforcer. Deux types de médiations s'offrent alors, l'une privilégiant le discours, l'autre le récit.

91 Dans ses Interviews imaginaires, Gide $^{37}$ invente un interviewer grâce auquel il peut se justifier de ses deux plus célèbres répliques, contestant l'une ( N'avez-vous pas écrit qu'avec les beaux sentiments, on fait de la mauvaise littérature? - On m'a suffisamment reproché cette phrase sans la comprendre, et, le plus souvent, en la citant tout de travers, oubliant au surplus que j'avais écrit tout de même La porte étroite ", p. 25); et réassumant l'autre ( Oserai-je vous demander pourquoi vous disiez naguère: «Victor Hugo, hélas $!^{38} "$ ? - Je le dirai encore. Si grande que soit mon admiration, je suis mal à l'aise dans son pathos ", p. 44).

92 Le droit ici exercé n'est pas un droit d'auteur, mais de simple locuteur. L'interview imaginaire déconstruit la vulgate citationnelle, dont nous avons vu la naissance en A1, avec les mémoires imaginaires, et l'achèvement en B1 avec le X par X des Ecrivains de toujours. 

naissant. Roland Barthes par Roland Barthes ${ }^{39}$ n'est ni une biographie autographe ni une autobiographie, mais un dictionnaire autocitationnel dans lequel les citations et leurs commentaires sont rangés dans un ordre alphabétique savamment perturbé. Alors qu’à la lettre B, l'article «Noms propres » désigne en creux le nom de l'auteur, c'est à la lettre A que celui-ci a placé son emblème: le Vaisseau Argo "dont les Argonautes remplaçaient peu à peu chaque pièce, en sorte qu'ils eurent pour finir un vaisseau entièrement nouveau, sans avoir à en changer le nom ni la forme » (p. 51). «Objet sans autre cause que son nom, sans autre identité que sa forme», obtenu par «substitution » et «nomination », la définition de l'objet mythologique est également la formule de ce témoignage autobiographique bien digne d'un sémioticien structuraliste. du jugement existentiel, est donc atteinte (C1). Elle a nécessité un minimum de leurres pré-attentionnels, quelques allusions autobiographiques pour Gide, une iconographie familiale limitée par Barthes à la période antérieure à l'entrée en écriture. Cet auteur n'est ni l'autorité vénérée par la postérité, ni la cause agissante : le scripteur, mais juste quelqu'un qui souhaite échapper à la chosification citationnelle et redevenir un interlocuteur.

\section{2 La relance narrative}

$\mathrm{Vu}$ leur position, entre titre et texte, Genette impute les épigraphes ${ }^{40}$ à l'auteur de fait, puisque de toute façon «il est aussi l'auteur du narrateur » et au narrateur en droit, puisqu'il parle au lecteur dans le texte. Elles exercent selon lui quatre fonctions: commentaire du titre, commentaire du texte, mise en valeur de l'auteur cité, mise en valeur de l'auteur citant. Néanmoins il nous semble que ce morcellement méconnait la fonction sémiotique de l'épigraphe qui est de corréler système intertextuel (auteurtexte cités- auteur- texte citants) et système intratextuel (titre -texte). Nous préférons parler de dominantes, l'une sémantique, l'autre symbolique, selon que l'épigraphe sollicite le rapport entre textes ou entre auteurs, en rappelant que, chaque fois, c'est l'intersystème complet qui est concerné.

Stendhal a intitulé le premier chapitre du livre II du Rouge et Le Noir « Les plaisirs de la campagne $»^{41}$. L'épigraphe, une citation de Virgile : « 0 rus quando ego te aspiciam!» reprend le sens du titre, en changeant de langue et de système énonciatif. La relation sémantique avec le chapitre est antonymique : Julien fuit Verrières et les balles de M. de Rênal, pour Paris où l'attend une place chez le marquis de La Mole. C'est donc Paris qu'il appelle de ses vœux et non la campagne. Un autre passager de la diligence fuit aussi « l'abominable vie que l'on mène en province » mais à regret, et rappelle à un ami qu'il "aime la fraîcheur des bois et la tranquillité champêtre ». Ce Parisien, apprend-on plus loin, avait acheté une terre près du Rhône dans les montagnes parce qu'il « avait soif de bonhomie et de simplicité ». Les querelles de clocher l'en ont chassé. On passe donc en quelques lignes d'une synonymie à une antonymie, les palinodies du comparse reflétant celle de Julien.

L'épigraphe fonctionne donc non comme un commentaire, ce que croit Genette, mais comme un emblème. C'est l'embrayeur ou représentamen d'une série d'interprétants qui peuvent concerner tout et/ou partie du texte, et qui tous comportent une part de ressemblance et une part de décalage (pouvant aller jusqu'à l'ironie) avec l'objet interprété : le titre.

Cahiers de Narratologie, 13 | 2006 

romanesques. Ils essaient d'écrire, en vain. Avec Lucien de Rubempré, Balzac décrit des débuts littéraires ordinaires. Les héros de Flaubert et de Joriff sont des Rubempré qui se seraient arrêtés à la déclamation de Chénier. Il est vrai que Lucien a commencé à écrire avant de découvrir Chénier, nuance capitale! La représentation donnée par ces personnages n'est pas celle d'un simple acteur de la république des lettres, mais d'un bagnard de l'écriture, image chère à Flaubert. Doubles de l'auteur, donc, dans ce qu'il a de plus tragique, son incapacité à être original. Les citations les habitent tellement qu'elles les abrutissent: "Pécuchet surtout fut ébahi par Génin. Comment! des $z^{\prime}$ hannetons vaudrait mieux que des hannetons? des z'aricots que des haricots?» (p. 247). Quand on sait le soin que Flaubert mettait à traquer l'assonance! 

de l'invention et de la théorie.

\section{4 La relance hypertextuelle} lettre supprimée : le « e ».

Lorsque Mops surgit de sa cave, on le croit idiot. Il croit simplement que tout le monde parle le Rabelais. Au stade précitationnel, le lecteur sauvage n'a pas encore conscience de ce que sont auteur, œuvre, et genre. Il lui faut d'abord découvrir un autre auteur, qui sera pour Mops Rousseau, dont il lit les Confessions, puis une autre œuvre du même auteur, les Rêveries, puis un autre genre, Littré, avant de pouvoir vraiment citer Rabelais. Car pour citer, il faut relire, et pour relire, il faut avoir oublié !

Assez proches de la métacommunication, ces doubles inférieurs de l'auteur mettent en scène les mille et un réflexes que doit acquérir un professionnel de l'écriture. Ils n'en appartiennent pas moins à la réalité imaginaire (C3). C'est justement pour cela qu'ils ont été conçus. Alors que le narrateur de la fiction autobiographique, proche de l'auteur, (Marcel dans La Recherche) révèle le côté exaltant de l'aventure littéraire, celui

Aussi étonnant que cela paraisse, la modification de l'intertexte ne produit pas toujours une dénudation des procédés d'écriture. Il suffit pour cela de bloquer les appels à la conscience métacommunicationnelle provoqués par la reconnaissance de l'hypotexte. Le moyen le plus simple est la dissimulation du mécanisme hypertextuel. Les cryptocitations de la Vie mode d'emploi sont un plagiat déclaré mais néanmoins réussi, le lecteur répugnant en général à interrompre sa lecture pour partir à leur recherche.

Deuxième cas de figure: la diversion comique. Il y a des transformations et des imitations dont le comique est intentionnel, ce sont les genres comiques: parodie, travestissement, pastiche héroï-comique, et d'autres dont le comique provient seulement de l'effet de surprise, distance et innocuité qu'elles provoquent et qui peut être involontaire. Dans La Disparition ${ }^{44}$, Perec soumet un corpus intertextuel à la contrainte fondatrice du roman, le lipogramme, qui est aussi le thème de l'histoire, puisqu'il s'agit de retrouver Anton Voyl, lequel n'est rien d'autre qu'une allégorie de la

Les "six madrigaux archi-connus qu'on a tous lus dans un Michard ou dans un Pompidou », transcrits de la main de Voyl sont censés fournir à ses amis « un jalon ». La lecture commence par Bris Marin de Mallarmus. Hugo est convoqué pour un Booz assoupi. Baudelaire désigné comme "un fils adoptif du commandant Aupick» vient ensuite avec trois "chansons": "Sois soumis mon chagrin, puis dans ton coin sois sourd» ("Sois sage, ô ma beauté et tiens-toi plus tranquille»), Accords (Correspondances) et Nos Chats (Les chats). Rimbaud clôt la série avec bien sûr " Vocalisations ».

Aucun de ces indices, pas même la reprise dans le sonnet de Rimbaud de «E blanc » par « un blanc » n'éclaire les héros qui en viennent à souhaiter l'aide d'« un Champollion », d'« un Chomsky», d'« un Roman Jakobson», "pourquoi pas un Oulipo?». Cette seconde série d'indices, qui en appelle directement au lecteur, n'a pas suffi, comme on le sait, à dessiller toutes les paupières. On peut en effet ne considérer que l'aspect fictionnel de la réécriture : le procédé de cryptage utilisé par Voyl, et/ou penser qu'on est devant un travestissement burlesque, puisque le thème lyrique des poèmes a été gardé, tandis que le style était abaissé.

Troisième cas de figure, l'effet de réel. L'aveu de l'hypertextualisation est parfois tellement imbriqué à la fiction que loin d'en altérer l'efficacité, elle la renforce. Ainsi les pastiches héroï-comiques auxquels Joriff s'est livré pour faire parler Frédéric Mops. Ainsi la réécriture homophonique d'un dicton épicurien dont Hugo fait dans Les 
misérables l'enseigne du cabaret Corinthe ${ }^{45}$. La spécialité de carpes au gras, orthographiée par son propriétaire "carpes hogras", est devenue, grâce aux intempéries « carpe horas».

110 Même sur un sujet sombre : la shoah, Perec dans $W$ ou le souvenir d'enfance ${ }^{46} \mathrm{n}$ 'a pas cru desservir son propos en obligeant le lecteur à refaire le douloureux va-et-vient entre les récits familiaux et l'écriture romanesque par lequel il s'était efforcé de remplacer sa mémoire disparue. On alterne les chapitres du récit autographe et ceux du roman décrivant une utopie nazie, sans pouvoir repérer les articulations qui permettraient de les reconstituer. Faisant réinstancier au lecteur ses propres comportements, Perec ne suscite pourtant pas la conscience métacommunicationnelle, mais bien l'adhésion fictionnelle (C4). En effet, il n'existe pas meilleur facteur de dissimulation des procédés que l'empathie.

111 Selon Marc Augé (op. cit., p. 78) « la forme rituelle emblématique du commencement ou du re-commencement serait l'initiation[...]. Ce qui s'efface et s'oublie alors, c'est simultanément celui que l'initié n'est plus et celui qu'il n'est pas encore, le même et l'autre en lui ». La formule convient parfaitement au visage de la citation que nous avons découvert dans cette partie. La citation donne au récit l'élan qui lui permet d'échapper aux codes, aux genres et aux stéréotypes. Elle est facteur d'inspiration, qu'elle paie d'ailleurs de sa disparition. Tout ceci ne va pas sans un certain brouillage des représentations. L'intertextualité finit par absorber les autres catégories genettiennes, et implose quand on tente d'analyser ses fonctions qui apparaissent totalement contradictoires. Quel signifié de puissance pourrait venir à bout d'équivalents sémantiques aussi hétéroclites qu' « imitation et invention », « imaginaire du palimpseste », "texte brisé », et "bricolage» (sous-titres du chapitre 3 «Esthétiques de l'intertextualité » de l'ouvrage cité de Piégay-Gros)?

Les trois entrées que nous avions choisies rendaient compte à la fois de la pratique experte des encodeurs : les stratégies ne sont pas les mêmes selon que l'on veut utiliser la citation dans un récit, faire un récit uniquement avec des citations ou se servir de la citation comme lanceur d'écriture; et des déformations prototypiques de la construction conceptuelle : chaque région occulte le fonctionnement des autres. Ainsi la citation du roman réaliste finit par assimiler l'intertexte à l'interdiscours, les centons ne gardent que son noyau citationnel, tandis que le tissage le noie dans le fonctionnement textuel.

113 A l'arrivée cependant, ce n'est pas trois mais quatre sorties qui s'offrent à nous.

114 En effet, reportés sur le tableau général obtenu en superposant les systèmes commandes et les systèmes états, les douze cas étudiés se répartissent selon quatre zones en fonction de leurs directions de contrôle :

-la zone 熙 regroupe citation reprise par le personnage a3, centon éditorial b1, et double naïf de l'auteur c3,

116 -la zone 媐 regroupe a1, a2, a4 (la presque totalité des citations reprises dans le récit) et le dialogue imaginaire $b 3$,

117 -la zone

118 -la zone ${ }_{60}^{60}$ regroupe centon hypertextuel b4, épigraphe c2, et relance hypertextuelle c4.

La bipolarité demeure : dans 臤 la narration sert à représenter le processus citationnel, c'est donc la citation qui oriente la narration; dans 敭 c'est l'inverse, la citation se dilue 


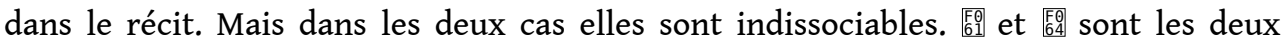

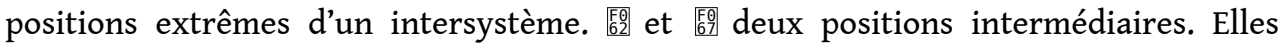
travaillent la portion de construction référentielle comprise entre vérité et fiction

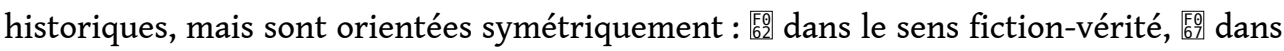
le sens vérité-fiction. Ce sont deux écosystèmes culturels différents. L'un, celui de 媐, est le vaste marché des biens symboliques dans lequel la citation s'échange comme une marque d'appartenance. L'autre, celui de 砲, est la loi citationnelle, défendue par ses gardiens, pourfendue par ceux qui n'ont pas envie d'être statufiés de leur vivant: les auteurs qui entendent garder le droit à leur parole.

Chaque zone représente une certaine approche de la citation: phénomène à

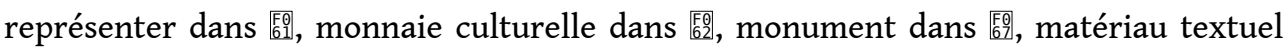
dans 熙. Mais c'est également un système dont l'équilibre repose sur des tensions. 盬 oppose à des représentations imaginaires: les personnages citateurs, une représentation de propos réels : le centon éditorial. 臨, en face des intégrations narratives, ménage l'issue du centon-dialogue imaginaire. En 䜿, recopiage externe et auto décentrement sont complémentaires, ils produisent en partant de la même base générique les figures inversées de la fausse interview et de l'interview imaginaire. Enfin dans l'aire de créativité qu'est 靄, l'épigraphe maintient l'ancrage historique de la citation face à des opérations qui le rejettent. Apparaissent ainsi les contours d'un écosystème qui pose, en face de chaque risque d'excès, un volant de rétablissement, qu'on pourrait appeler aussi «déviation correctrice». Celle-ci a pour fonction de réactiver un aspect de la réalité que la pratique dominante dans la zone tend à

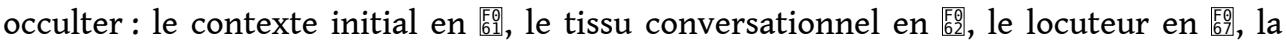
valeur symbolique de la mémoire en 蕅.

Le détail des analyses et la cartographie finale ont montré également que l'intersystème comporte des régulations internes à différents niveaux, et parfois même plusieurs régulations pour le même problème. Ainsi la maquette éditoriale X par X par Z (b1) est transgressée aussi bien par le Barthes par Barthes (c1) que par le Ronceraille (b4), pour des motifs différents- Barthes était à la fois juge et partie (critique et auteur célèbre), Claude Bonnefoy seulement critique-, mais avec un mobile identique : tordre le cou au mythe de l'auteur. Quant à l'«idiot citateur", on pourrait y voir une manifestation d'auto- ironie du romancier fatigué du double registre citationnel: narrateur et personnage, imposé par le pacte réaliste.

On notera aussi l'importance de l'écriture de centon (b), inversement proportionnelle à la reconnaissance culturelle qui lui est généralement octroyée. Non seulement elle réveille la conscience du lecteur par l'impression d'étrangeté qu'elle procure, même quand l'intertexte est totalement dissimulé, mais surtout elle joue un rôle clef dans la formation de la personnalité auctoriale. De certains enfants qui éprouvent de la difficulté à communiquer, le psychiatre Boris $\mathrm{Cyrulnik}^{47}$ remarque qu'ils ne se libèrent que dans la pratique théâtrale parce que les dialogues leur sont imposés, qu'ils n'ont pas à en prendre l'initiative. De même, certains auteurs ne peuvent atteindre le récit que par le détour intertextuel. C'est le cas, d'ailleurs signalé par Cyrulnik et pour les deux ouvrages que nous avons évoqués : La disparition et $W$, de George Perec, resté sans « voix » autobiographique.

Grâce au mais aussi au delà du discours, narration et citation participent à la construction de l'identité des hommes et des cultures. "L'identité humaine est essentiellement narrative» dit Cyrulnik (p.159), nous ajouterons qu'elle est aussi 
intertextuelle. Que récit et intertexte soient authentiques ou fictionnels, la narration sans la citation court le risque de se refermer sur elle-même, de se perdre dans le fantasme intérieur de ces sujets qui ne confrontent jamais leur scénario à la version d'autrui.

\section{NOTES}

1. Le démon de la théorie, Littérature et sens commun, Seuil, 1998.

2. 1968, repris dans Le bruissement de la langue, 1984.

3. Dunod, 1996.

4. Cf. Dominique Maingueneau, L'Analyse du discours, Hachette, 1991, p. 20.

5. Grammaire du sens et de l'expression, Hachette, 1992, p. 649.

6. P. 650 : «Cet acte d'énonciation qui décrit « la relation à l'autre-tiers » est en fait un peu particulier. On sait, en effet, que tout acte de langage dépend d'une façon ou d'une autre du sujet parlant et de ses différents points de vue. Il s'agit donc ici d'un « jeu » que se joue le sujet parlant, comme s'il lui était possible de ne pas avoir de point de vue, de disparaitre complètement de l'acte d'énonciation, et de laisser parler le discours par lui-même ».

7. Encyclopaedia of mathematics, Kluwer academic publishers, Dordrecht/Boston/ London, p. 886 : « Control system ». La théorie du contrôle présuppose la théorie des systèmes mais s'en distingue parce qu'elle ne s'intéresse qu'à leurs caractéristiques fonctionnelles sans tenir compte de leurs propriétés internes. De ce fait, elle peut considérer comme identiques deux systèmes différents par leurs caractéristiques mais qui ont le même comportement et les mêmes buts (« two control systems, having in some sens identical behaviour and identical purposes, are not regarded as distinct »). Ce qui explique en particulier que deux textes appartenant à des genres d'écrits différents puissent avoir à certains moments un effet identique sur le lecteur, parce qu'ils utilisent les mêmes variables de contrôle et produisent les mêmes effets. Ce phénomène bien connu des théoriciens de la littérature a désespéré de tous temps ceux qui ont essayé d'établir une typologie des genres, et n'est pas vraiment résolu par la notion de « dominante » introduite par les formalistes russes pour assouplir la classification générique.

8. Seuil, p. 263. S'inspirant du modèle jakobsonien, Genette distinguait cinq fonctions : narrative (référentielle), régie (métalinguistique ou métanarrative), communicative (phatique et conative), testimoniale (émotive et idéologique). Reuter, Analyse du récit, Nathan, 2000, p. 43, distingue fonction de régie et fonction métanarrative, dont il fait une sorte de super-régie : « fonction de régie explicite qui consiste à commenter le texte en signalant son organisation interne ». En trente ans, le modèle a évolué vers une spécification par actes de langage : modalisation, évaluation, explication.

9. Jean-Marie Schaeffer, Pourquoi la fiction?, Seuil, 1999, p. 123.

10. Genette, Seuils, 1982, p. 14.

11. Logique des genres littéraires, Seuil, 1986, insiste sur l'atemporalité de ce discours, p. 82. 
12. Les formes de l'oubli, Payot, Rivages, 2001, p. 77.

13. Livre de Poche, Plon, 1951, p. 423.

14. Cf. Gilles Thérien, « Pour une sémiotique de la lecture », Protée, print. 90, p. 77.

15. Pierre Loti, Bookking international, 1994, Paris, p. 23.

16. Macula, 1990, préface de P. et R. Wald Lasowski, p. 7.

17. J.- Fr. Labie, « Verdi et la politique, un malentendu qui dure », Viva Verdi, Télérama, janv. 2001, p. 41.

18. La Comédie humaine, tome 3, Seuil, 1966, p. 396 .

19. Ibid. : « Lucien lut à son tour l'épique morceau de l'Aveugle et plusieurs élégies.

Quand il tomba sur le fragment : S'ils n'ont point de bonheur, en est-il sur la terre? il baisa le livre, et les deux amis pleurèrent, car tous les deux aimaient avec idolâtrie ».

20. GF Flammarion, 1990, p. 106.

21. Sur cette notion, qui approche le souvenir en creux, comme une absence évoquée par la trace mais non récupérable, voir J. -B. Pontalis, Ce temps qui ne passe pas, Gallimard, 1997.

22. Lucien est censé avoir écrit en province ces vers parus dans les Annales romantiques de 1828, op. cit., p. 405, et p. 417, et d'autres écrits en 1824 par Balzac pour Julie Campi, fille de Mme de Berny.

23. Op. cit., pp. 466-467, l'attribution des sonnets est donnée par Pierre Citron dans une note. Par ailleurs Colette rappelle l'anecdote et cite le sonnet de Gautier dans le portrait qu'elle consacre à « La Tulipe » (Pour un herbier, Mermot, Cercle du bibliophile, Lausanne, s. d., p. 38).

24. Dargaud, 1979 .

25. Op. cit., pp. 225. Cette relecture est en fait une restitution car Hugo avait déjà convoqué Rome...comme comparé : « Et, lanciers, grenadiers aux guêtres de coutil,/ Dragons que Rome eût pris pour des légionnaires/ Cuirassiers, canonniers qui traînaient des tonnerres/ Portant le noir colback ou le casque poli/ Tous ceux de Friedland et ceux de Rivoli ». Goscinny opère donc une traduction-retour : "Triarii, principes aux caligae de cuir,/ Hastati dont Rome faisait des légionnaires,/ Vélites, sagittarii qui traînent leur crinière, portant des clipeus et jambières de métal/ Tous ceux d'Alésia et ceux de Pharsale ».

26. La seconde main, ou le travail de la citation, Seuil, 1979, p. 15.

27. Gallimard, 1970.

28. Seuils, Seuil, 1987.

29. Cité par Jean Bruneau dans sa préface au tome 1 de l'édition Pléiade de la Correspondance, 1973, p. XXX.

30. B. Dupriez, Gradus, 10/18, UGE, 1984, p. 364.

31. Télérama, $\mathrm{n}^{\circ}$ cit. , pp. 27-30.

32. «Cette interview est réalisée à l'aide de la correspondance de Verdi et de propos qu'il a réellement tenus. Seules, parfois, ont été modifiées la ponctuation et la concordance des temps ".

33. Alphonse Allais, Euvres posthumes, Laffont, Bouquins, pp. 259-60, et 262-63. Sam Weller est le domestique de M. Pickwick dans le roman de Dickens.

34. Belfond, 1979.

35. Gallimard, La noire, 1998.

36. Claude Bonnefoy, Ronceraille, Seuil, 1978.

37. Gallimard, 1942.

38. Réponse à la question « Quel est le plus grand poète français ?». 
39. Seuil, 1975.

40. Seuils, pp. 134-149.

41. Livre de Poche, 1958, p. 235.

42. Euvres complètes, t. 2, Seuil, 1964.

43. Bourin, 1987.

44. Denoël, 1969, p. 118. Sur la réécriture des intertextes dans La disparition, on consultera dans Littératures $n^{\circ} 7$, printemps 1983, l'article de John Lee, « Brise ma rime », pp. 11-21.

45. Troisième partie, livre XII, Nelson, p. 423.

46. Denoël, 1975.

47. Les vilains petits canards, Odile Jacob, 2001.

\section{RÉSUMÉS}

Par le concept d'« intertexte » le structuralisme prenait acte de la double présence dans le texte de l'histoire et de la parole. Mais cette dimension était trop proche de l'approche traditionnelle de la critique des sources pour ne pas être frappée de suspicion culturelle. Seule la critique de la réception s'y est attachée pour conclure un peu vite à l'impossibilité de la circonscrire. Le temps est donc venu de s'intéresser à l'interaction auteur-lecteur dans la communication intertextuelle. Ce secteur pouvait paraître secondaire à la narratologie tant qu'elle restait occupée des structures internes du récit. C'est la pragmatique textuelle qui, en explorant les conditions réelles de son énonciation, a redonné à la relation du récit et des discours toute son actualité.

La théorie du contrôle fait apparaître trois champs d'interaction entre acte narratif et acte citationnel : celui où domine la narration est lié à la remémoration, celui où domine la citation est lié à l'occupation du présent par le passé, celui où citation et narration se croisent est lié au réinvestissement du passé dans le futur. Les auteurs organisent leurs stratégies autour des rôles sociaux de la citation : événement, monnaie d'échange culturelle, monument, matériau sémiotique. Dans, et entre chacune de ces zones de choix, les déplacements sont facilités par des « déviations correctrices » qui empêchent l'interaction de se bloquer sur un rôle.

INDEX

Mots-clés : théorie

\section{AUTEUR}

NICOLE BIAGIOLI

Université de Nice-Sophia Antipolis 